\title{
Selective backscattering and the breakdown of the quantum Hall effect
}

\author{
L.W. Molenkamp, M.J.P. Brugmans ', H. van Houten, C.W.J. Beenakker \\ Philips Research Laboratones, $5600 \mathrm{JA}$ Eindhouen, Netherlands
}

and

C.T. Foxon

Phllps Research Laboratones, Redhil, Surlcy, RHI 5HA, UK

Received 4 June 1991; accepted for publication 26 August 1991

We study the breakdown of the quantum Hall effect in a natrow channel using quantum point contacts as edge channel mixers, and as voltage piobes. We observe a dependence of the two-terminal and Hall resistances in the breakdown regime on the adjustment of the point contacts, in a manner which demonstiates selective backscatteing in the highest occupied Landau level An extension of Buttıker's theory of the quantum Hall effect with a Hall-voltage dependent backscattering rate accounts for some of our observations

The breakdown of the quantum Hall effect at high current densities (the regime of non-linear response) still is not very well understood [1-3]. Several mechanisms have been proposed (see ref. [3] for a recent discussion), but the interpretation of the experiments is not unambiguous. Experimentally, the breakdown is conveniently studied [2] in a narrow $(\sim 1 \mu \mathrm{m})$ channel or constriction. In such structures large Hall fields can be generated at moderate current levels $(\sim 0.1-1 \mu \mathrm{A})$. Recently, we have reported [4] results of an experimental study of the breakdown of the quantum Hall effect in a novel geometry, i.e., a narrow channel fitted with adjustable point contact voltage probes. We use the voltage probes as edge channel mixers [5], to regulate the equilibration of the highest occupied Landau level with the lower levels $[5,6]$. This technique has enabled us

\footnotetext{
${ }^{1}$ Also at. Eindhoven Univeisity of Technology, $5600 \mathrm{MB}$
} Eindhoven, Nethet lands to demonstrate that breakdown occurs predominantly through selective backscattering of electrons in the highest Landau level [4]. In our previous report, we discussed predominantly results on the four-terminal longitudinal resistance of the channel. Here, we present supplementary data on the two-terminal and Hall-resistance, and compare these experimental results with a model based on Büttiker's theory of the quantum Hall effect [7], extended to the non-linear regime.

The top of fig. 1 gives a layout of the structure used in this work. The sample is fabricated from a high mobility $(\mathrm{Al}, \mathrm{Ga}) \mathrm{As}$ heterojunction wafer containing a $2 \mathrm{DEG}$ with a sheet electron concentration $n_{\mathrm{s}}=3.5 \times 10^{11} \mathrm{~cm}^{-2}$ and a mobility $\mu=$ $1.4 \times 10^{6} \mathrm{~cm}^{2} / \mathrm{V} \cdot \mathrm{s}$. In the figure, crosses indicate ohmic contacts to the 2DEG; the hatched areas are split gates that are used to electrostatically define a channel of width $W=4 \mu \mathrm{m}$ and length $L=18 \mu \mathrm{m}$. Two opposite pairs of quantum point contacts are defined on the top $\left(t_{1}\right.$ and 

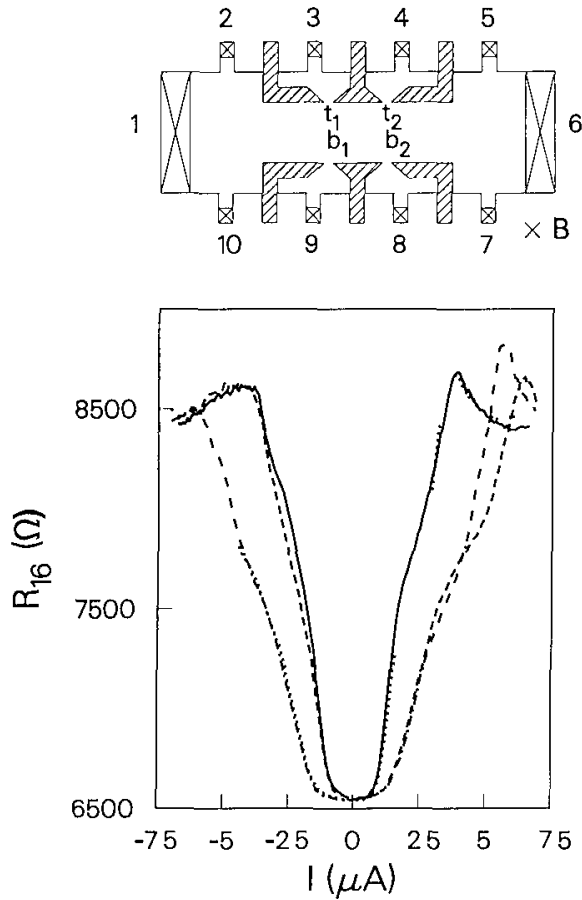

Fig 1 Top lay-out of the Hall-bar (not to scale), containing a narrow channel (of width $4 \mu \mathrm{m}$ and length $18 \mu \mathrm{m}$ ) with point contact voltage ptobes ( $3 \mu \mathrm{m}$ apart) Positive current flows from ohmic contact 1 to 6 Bottom two-termmal differential iesistance $R_{16}$ vetsus current for four different configurations of the point contact voltage probes $\left(N_{1}, N_{\mathrm{b}}\right)=(2,2)$ (sold curve), $(1,2)$ (dashed), $(2,1)$ (dotted), and $(1,1)$ (dash-dotted)

$\left.t_{2}\right)$ and bottom $\left(b_{1}\right.$ and $\left.b_{2}\right)$ edge of the channel, with a separation of $3 \mu \mathrm{m}$ between adjacent point contacts. The gate voltages are adjusted such that adjacent point contacts have equal resistance $\left(R_{\mathrm{t}_{1}}\right.$ $=R_{\mathrm{t}_{2}} \equiv R_{\mathrm{t}}$ and $R_{\mathrm{b}_{1}}=R_{\mathrm{b}_{2}} \equiv R_{\mathrm{b}}$ ). We present results obtained at a temperature of $1.65 \mathrm{~K}$, and a magnetic field $B=3.45 \mathrm{~T}$, corresponding to a filling factor $\nu=n_{\mathrm{s}} h / 2 \mathrm{eB}=2.0$ in the narrow channel. (Because of electrostatic depletion, $n_{\mathrm{s}}$ in the channel is somewhat smaller than in the bulk 2DEG, where $\nu=2.1$ at 3.45 T.) A current $I$ is passed through the channel from ohmic contact 1 to 6 . With the magnetic field direction as indicated in the figure, and for positive currents, the top edge of the channel has the highest electrochemical potential (i.e., it is charged negatively). The differential resistance between ohmic contacts $l$ and $\jmath, R_{l j}=\mathrm{d} V_{l j} / \mathrm{d} I$, with $V_{l j} \equiv V_{l}-V_{\jmath}$, is measured using a low-frequency lock-in technique. Data have been obtained for four different sets of values of the point contact resistances $R_{\mathrm{t}}$ and $R_{\mathrm{b}}$. These sets correspond to different numbers $\left(N_{t}, N_{b}\right)$ of spin-degenerate edge channels that are fully transmitted through the point contacts on either side of the channel (note that $R_{\mathrm{t}, \mathrm{b}}=h / 2 e^{2} N_{\mathrm{t}, \mathrm{b}}$ ). The configurations used are $\left(N_{\mathrm{t}}, N_{\mathrm{b}}\right)=(2,2),(1,2),(2,1)$, and $(1,1)$.

In the lower panel of fig. 1 we show the current dependence of the differential two-terminal resistance of the channel $\left(R_{16}\right)$. Beyond a current of 1-2 $\mu \mathrm{A}, R_{16}$ suddenly increases, indicating the onset of breakdown. Clearly, the adjustment of the point contacts at the channel boundaries strongly influences the breakdown characteristics. When the point contacts transmit all edge channels $\left(\left(N_{\mathrm{t}}, N_{\mathrm{b}}\right)=(2,2)\right.$, solid curve $)$, the breakdown occurs at a relatively small current. However, when the highest occupied edge channel is reflected $\left(\left(N_{\mathrm{t}}, N_{\mathrm{b}}\right)=(1,1)\right.$, dashdotted), considerably larger currents are required to obtain breakdown. For positive currents, the breakdown curves for the mixed sets $\left(\left(N_{\mathrm{t}}, N_{\mathrm{b}}\right)=\right.$ $(1,2)$, dashed and $(2,1)$, dotted) coincide with the curves for the symmetric sets $(2,2)$ and $(1,1)$, respectively. For negative currents, this correspondence is reversed. This implies that the breakdown characteristics are affected only by the adjustment of the voltage probes on the high-potential edge.

The effects of the adjustment of the point contacts on the breakdown can be understood qualitatively, assuming that backscattering occurs only in the upper Landau level, and that interLandau level scattering is negligible. Experimental support for both assumptions has been given in ref. [4]. The argument then is the following. Electrons entering the narrow channel along the top edge (assume positive current) in the highest Landau level are backscattered due to the proximity of the edge channel at the opposite edge. Further down the channel, a steady-state situation is reached, but when the highest Landau level is transmitted through the point contact at the high potential edge $\left(N_{\mathrm{t}}=2\right)$, it is equilibrated with the other edge channels. This causes a repopulation of the partially depleted highest level, 


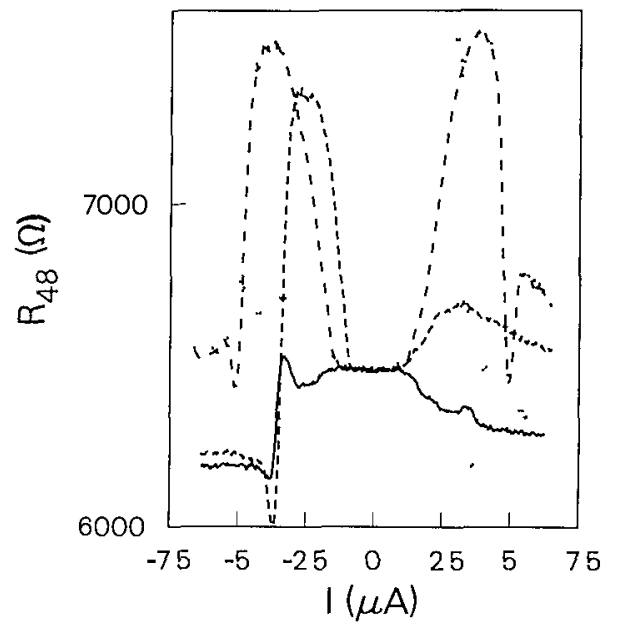

Fig 2 Differential Hall resistance $R_{48}$ versus current for the same point contact configurations as in fig 1 (with the same coding of the curves)

and consequently a second opportunity for backscattering.

Fig. 2 shows the results of our experiments on the differential Hall resistance $R_{48} \equiv \mathrm{d} V_{48} / \mathrm{d} I$, measured using one of the quantum point contacts at the top (4) and one at the bottom (8) edge of the channel as voltage probes. In these data, we find a strongly enhanced breakdown when the point contact at the low potential edge is adjusted such that the highest occupred edge channel is not transmitted. We interpret this effect as a manifestation of the anomalous integer quantum Hall effect $[6,8,9]$. An anomalous Hall resistance is known to occur as a result of non-equilibrium population distribution of the edge channels, provided that at least one of the voltage probes used in the measurement is non-ideal (in the sense that not all edge channels are fully transmitted). In our experiment the current contacts are ideal, and the presence of a non-equilibrium population of the edge channels (essential for the observed anomalous behaviour) must be the result of selective backscattering in the channel. This observation supports our earlier conclusion [4] that breakdown occurs selectively in the highest Landau level, and is consistent with our interpretation of the two-terminal data, dis- cussed above. For strongly positive currents, $R_{48}$ as measured for the pairs $(1,1)$ and $(2,1)$ is suddenly reduced to values comparable to $R_{48}$ for the pairs $(1,2)$ and $(2,2)$. (At strongly negative currents the same effect occurs for $(1,1)$ and $(1,2)$.) We attribute this to the onset of interLandau level scattering at the low-potential edge of the channel. A similar breakdown of adiabaticity has been observed in studies of the anomalous quantum Hall effect in wide conductors [8].

We have attempted to model our observations starting from Büttıker's description [7] of the quantum Hall effect in linear response. To account for the non-linearities, we have used an energy dependent backscattering probability $r(E)$ which depends on the Hall voltage $V_{\mathrm{Hall}}$ in a self-consistent manner. This is illustrated schematically in fig. 3 , which depicts the variation of the energy of the highest Landau level along a cross section of the narrow channel at finite positive current [3], so that the electrochemical potential of the top edge $\left(\mu_{\mathrm{t}}\right)$ is increased by $\mathrm{eV}$ Hall with respect to that of the bottom edge $\left(\mu_{b}\right)$. Occupied states are denoted by a thick line for electrons with a negative group velocity, and by a dashed line for electrons with a positive group velocity. The bottom of the Landau level has energy $E_{0}$, which is independent of $V_{\mathrm{Hall}}$. Fig. 3 illustrates that for electrons with energy $E$ between $\mu_{\mathrm{b}}$ and $E_{0}+e V_{\text {Hall }}$, the distance between the high- and low-potentral edge can be cont1nously dimınıshed by increasing $V_{\mathrm{Hall}}$. We assume

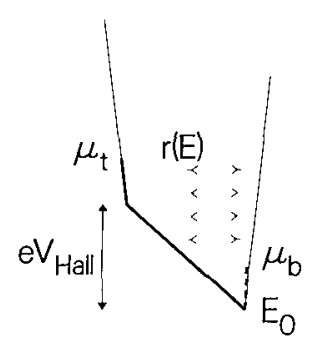

Fig 3 Schematic energy diagram of the highest occupied Landau level, tilted due to the Hall voltage $V_{[1,1]}$ along a cioss section of the nar row channel 


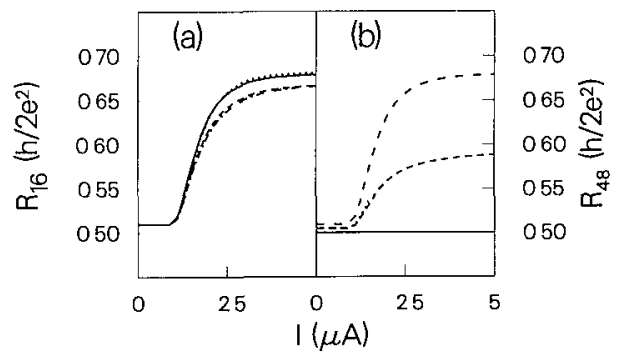

Fig 4. Results of our calculations for $R_{16}$ (a) and $R_{48}$ (b). The coding of the curves is the same as in figs. 1 and 2 .

that backscattering in the highest level decreases exponentially with this distance, according to:

$$
\begin{array}{rlrl}
r(E)=0 & \text { for } E \leq E_{0}, \\
=K \exp \left[-2\left(E-E_{0}-e V_{\text {hall }}\right) / e V_{\text {Hall }}\right] \\
& \quad \text { for } E_{0}<E \leq E_{0}+e V_{\text {IIall }}, \\
=K & \text { for } E>E_{0}+e V_{\text {IIall }}
\end{array}
$$

where $K$ is a constant. We neglect backscattering in the lower Landau level. The above expression for $r(E)$ is incorporated in a set of LandauerBüttiker equations describing a channel with two ideal current contacts, and two opposite point contact voltage probes, which are solved for the probe potentials. Since $V_{\text {Hall }}$ and $r(E)$ are mutually interdependent, the calculation is iterated until a self-consistent result is obtained.

Results of our calculation for the differential resistances $R_{48}$ and $R_{16}$ are shown in fig. 4, for positive currents, and for filling factor $\nu=2.0$. The value of $K=0.067$ was chosen to yield approximately correct values for the differential resistance. The calculations for the two-terminal resistance $R_{16}$ in fig. 4a exhibit for low currents a flat "quantized" region. In this region $R_{16}$ is not exactly equal to $h / 4 e^{2}$, because of the finite backscattering probability $\left(r\left(E_{\mathrm{F}}\right) \sim K\right)$ in the narrow channel for energies near the Fermi level ( $E_{\mathrm{F}} \approx \mu_{\mathrm{b}} \approx \mu_{\mathrm{l}}$ for low currents). The resistance starts rising steeply when $e V_{\text {Hall }} \approx \mu_{\mathrm{b}}-\mathrm{E}_{0} \approx$ $\hbar \omega_{\mathrm{c}} / 2$ (threshold of breakdown), and saturates for currents where the backscattering probability approaches $K \exp (2) \approx 0.5$ over a wide energy range, as follows from eq. (1) in the limit that $V_{\text {Hall }} \gg E, E_{0}$. Our calculations for $R_{16}$ yield a similar pairing of the breakdown curves as the experimental data, reflecting the importance of the top edge voltage probes. The calculated Hall resistance $R_{48}$ also agrees qualitatively with the experiment: the trace for $\left(N_{\mathrm{t}}, N_{\mathrm{b}}\right)=(1,1)$ shows the strongest anomalous resistance peak; the anomaly is somewhat smaller for $(2,1)$, and smaller still for $(1,2)$. For $\left(N_{\mathrm{t}}, N_{\mathrm{b}}\right)=(2,2)$ no deviations from quantization occur, the reason being that the voltage probes used to measure the Hall resistance are ideal in this case.

Some notable discrepancies between calculation and experiment remain. Breakdown is found experimentally to occur at different current levels. In the calculation for the two-terminal resistance, however, the curves start to deviate from quantization at the same current level. For the Hall resistance, the experimental $(2,2)$ curve shows resistances reduced below the quantized value, whereas the calculation shows no breakdown at all. Also the reduction of $R_{48}$ at large currents for the $(1,1),(1,2)$ and $(2,1)$ configurations is not reproduced by the calculation.

In conclusion, our new experimental results support the idea proposed in ref. [4] that breakdown of the quantum Hall effect in a narrow channel proceeds predominantly via selective backscattering within the highest Landau level. Modelling the breakdown phenomena with a Hall-voltage dependent backscattering probability $r(E)$ yields a reasonable qualitative description of some characteristic features of the experiments, but other features call for a less simplified model (which presumably should also include interLandau level scattering).

The authors would like to thank B.W. Alphenaar, P.C. van Son and A.A.M. Staring for useful discussions, and M.A.A. Mabesoone and C.E. Timmering for expert technical assistance. We acknowledge the stimulating support of M.F.H. Schuurmans, and partial funding under the ESPRIT basic research action project 3133 .

\section{References}

[1] G. Ebert, K. von Klitzıng, K. Ploog and G. Weimann, J. Phys C (Solid State Phys.) 16 (1983) 5441, 
M E Cage, R F Dzluba, B F Field, E R Willıams, S M Girvin, A C Gossard, D C Tsuı and R J Wagner, Phys Rev Lett 51 (1983) 1374

[2] L Bhek, E Braun, G Hein, V Kose, J Niemeyer, G Weimann and $\mathrm{W}$ Schlapp, Semicond Scı Technol 1 (1986) 110 ,

J R Kirtley, Z Schlesinger, T N Theis, F P Milliken, S L Wright and LF Palmateer, Phys Rev B 34 (1986) 5414, PGN de Vegvar, AM Chang, G Timp, PM Mankiewich, JE Cunningham, R Behrunger and RE Howard, Phys Rev B 36 (1987) 9366

[3] P C van Son, GH Kruithof, and T M Klapwuk, Phys Rev B 42 (1990) 11267

[4] LW Molenkamp, M J P Brugmans, $H$ van Houten,
C W J Beenakker and C T Foxon, Phys Rev B 43 (1991) 12118

[5] B J van Wees, LP Kouwenhoven, EMM Willems, C J P M Harmans, JE Mooij, $H$ van Houten, CW J Beenakker, J G Willamson and C T Foxon, Phys Rev B 43 (1991) 12431

[6] B J van Wees, E M M Willems, C J P M Harmans, C W J Beenakker, $H$ van Houten, J G Willamson, C T Foxon and J J Harris, Phys Rev Lett 62 (1989) 1181

[7] M Buttıke1, Phys Rev B 38 (1988) 9375

[8] S Komiyama, H Hird1, S Sasa and S Hiyamızu, Phys Rev B 40 (1989) 12566

[9] B W Alphenaar, P L McEuen, R G Wheeler and R N Sacks, Phys Rev Lett 64 (1990) 677 